\title{
The polypill and the prevention of heart attacks and strokes
}

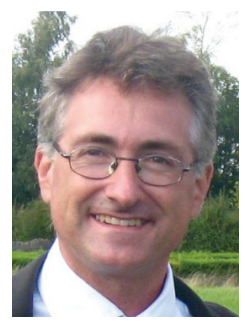

\section{David S Wald}

Centre for Environmental \& Preventive Medicine, Wolfson Institute of Preventive Medicine, Barts \& The London, Queen Mary's School of Medicine \& Dentistry, London, EC1M 6BQ, UK =d.s.wald@qmul.ac.uk

\section{"The polypill is more than just a pill. It is a strategy for preventing cardiovascular disease."}

David S Wald speaks to Caroline Telfer, Assistant Commissioning Editor. David S Wald is a Consultant Cardiologist and Reader in Preventive Cardiology. He trained at Oxford University (UK) and Imperial College of Science and Technology, London (UK). His work combines interventional and preventive approaches to cardiovascular disease. He is currently leading a multicenter randomized trial assessing the value of preventive angioplasty in patients with acute myocardial infarction and a UK trial of a polypill for people over the age of 50 years for the prevention of ischemic heart disease and stroke.

\section{What attracted you to a career in cardiac medicine?}

The realization that it was possible to fix people. At the time I chose my career, there were few specialties other than cardiology in which it was clear that the treatments really worked and could change people's lives; for example, angioplasty, pacing, treatment for heart attacks and even heart failure. This, taken together with the fact that heart disease and stroke were the most common causes of death and disability, made it an extremely exciting field to get into. My interests are now in percutaneous coronary intervention and prevention: two areas of cardiology that are often viewed as distinct, but in fact are quite complementary.

\section{In your opinion, what are the most important issues in cardiology that should be tackled in the near future \& how might this happen?}

The prevention of heart attacks and strokes. Smoking, the increase in dietary fat and salt, and diabetes have collectively caused an epidemic of cardiovascular disease over the last hundred years. Because much of this is man-made, most cardiovascular disease events can be avoided. While there has been a substantial decline in the agespecific mortality of cardiovascular disease over the last 20 years or so, the total number of deaths has not changed much: people are getting the disease, just when they are a bit older. Effective treatments to prevent heart disease and stroke are already available. The problem is that we are not using them as effectively as we could. The combination of lifestyle measures, legislation to improve the content of our food supply, together with the sensible use of preventive medication, could make the most common cause of death and disability quite rare, even in our lifetime. But it will take a concerted effort on the part of doctors, the public and healthcare organizations to achieve this.

- In recent years you \& your team have been pioneering the polypill for the prevention of cardiovascular disease in older adults. Can you explain a little about the details of this medication?

The polypill is more than just a pill. It is a strategy for preventing cardiovascular disease. The strategy is based on the recognition that lowering causal cardiovascular risk factors, such as cholesterol and blood pressure (BP), from whatever level they start at, is beneficial and produces the same proportional reduction in risk of disease for a given change in the risk factor. This means that interventions to reduce causal risk factors should be offered to all people who are at high risk of a heart attack or stroke, regardless of the level of the risk factor. A person's age is the simplest and strongest determinant of risk, and is therefore a simple, fair and inexpensive means of selecting people for preventive treatment. The underlying pharmacological concept behind the polypill is to combine drugs that intervene on the principal causal pathways of ischemic heart disease and stroke (a statin and three BP-lowering drugs), at doses that minimize adverse effects and maximize efficacy.

\section{- You mentioned the components of the polypill. How did you decide upon these components?}

The components of our polypill include a statin at full dose and three BP-lowering drugs, each at half-standard dose. The reason for using different classes of BP-lowering drugs in combination is that they act additively in lowering BP.

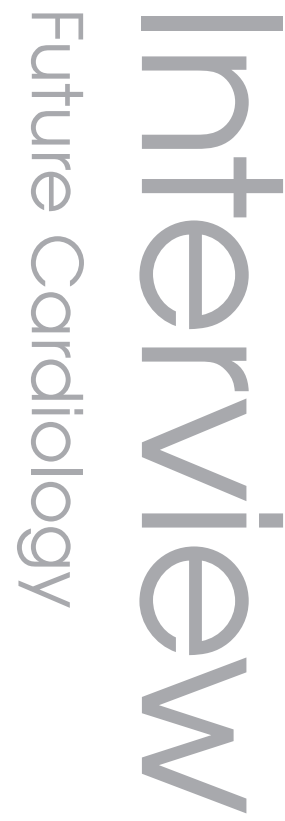

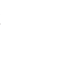


Combining two drugs from different classes is approximately five-times more effective than doubling the dose of one. By using half-standard doses you minimize adverse effects. The decision to use the specific drugs (losartan $25 \mathrm{mg}$, hydrochlorothiazide $12.5 \mathrm{mg}$ and amlodipine $2.5 \mathrm{mg}$, together with simvastatin $20 \mathrm{mg}$ ) was based on balancing efficacy and safety, and ensuring that we had freedom to operate because some drugs or drug combinations are on patent. All of the drugs we are using are generic, which means that they are also affordable.

- You mentioned that they would be used in older adults, what exact demographic will this be aimed at \& how did you decide upon this? Healthy adults aged 50 or over. Approximately 95\% of all heart attacks and strokes happen over the age of 50 years, so offering a polypill that could prevent approximately two-thirds of all heart attacks and strokes to everyone in the age group when these disorders occur ensures that almost everyone who stands to benefit will benefit.

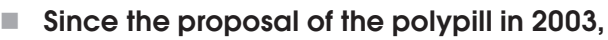 what progress has been made?}

We have developed a polypill with the help of Cipla (Mumbai, India), assessed it in a randomized controlled trial and showed that it has the expected effects on lowering BP and cholesterol [1].

\section{- Could you give an overview of the main findings of the recent clinical trials in a bit more detail?}

Our trial was published in PLoS ONE in 2012. It was a randomized controlled crossover trial in which every participant acted as their own control; everyone received the polypill for 12 weeks and a placebo for 12 weeks with the sequence of polypill and placebo determined randomly [1]. This design enabled us to determine, with only a small number of subjects $(n=86)$, an accurate estimate of the effect of the polypill on lowering $\mathrm{BP}$ and LDL cholesterol. The reductions were almost exactly as predicted: we observed a $12 \%$ reduction in $\mathrm{BP}$ and a $39 \%$ reduction in LDL cholesterol.

\section{- Was there anything you saw during these trials that you didn't expect?}

Initially, we were surprised that the results were so close to expectation. But on reflection, it is perhaps not that surprising because the predictions themselves were based on studies of data from hundreds of thousands of people from many trials and cohort studies, assessed together in a meta-analysis, and there was no reason to expect that these analyses were wrong.

\section{- What are your views on the use of the} polypill as a matter of primary prevention?

I'm supportive. It should, of course, be used in conjunction with a sensible diet and lifestyle. In other words, the polypill is not a replacement for moderating the intake of saturated fat, avoiding smoking and dietary salt, limiting alcohol consumption and taking regular exercise. All of these things are important and should happen as part of a normal life. The problem is that it is extremely hard to achieve this sort of 'normality' in modern society and, therefore, almost impossible to attain the degree of BP and cholesterol lowering needed to avoid a heart attack and stroke. The polypill offers, in a simple and safe way, the means of achieving these large reductions and, if used widely and with a high level of adherence, could avoid most cases of heart attacks and stroke in the population.

\section{- Are there any medications that are similar?}

The individual components of the polypill are all available, but you would have to break two of the BP-lowering drugs in half because they are not available at the low doses required. With a bit of hassle, however, you could take the ingredients and you would expect to achieve the same benefits.

- You mentioned that this has already been through recent clinical trials. What are the next steps for the medication?

To make the polypill available and readily accessible to the public.

\section{Disclaimer}

The opinions expressed in this interview are those of the interviewee and do not necessarily reflect the views of Future Medicine Ltd.

Financial \& competing interests disclosure

DS Wald undertakes research on a polypill for the prevention of cardiovascular disease and has an interest in its development. DS Wald has no other relevant affiliations or financial involvement with any organization or entity with a financial interest in or financial conflict with the subject matter or materials discussed in the manuscript apart from those disclosed.

No writing assistance was utilized in the production of this manuscript.

\section{Reference}

1. Wald DS, Morris JK, Wald NJ. Randomized polypill crossover trial in people aged 50 and over. PLoS ONE 7(7), e41297 (2012). 